of Richmond Health Department, and Dr. I. Taylor and Dr. T. Sebestyen of Ealing Health Department. We are indebted to all the general practitioners in the three boroughs for their close co-operation, so vital to the survey. We thank Dr. Hillas G. Smith of Neasden Hospital, Dr. P. L. N. Baly of St. John's Hospital, Uxbridge, and Dr. J. Vahrman of Western Hospital, Fulham, who kindly provided access to patients, Mr. T. G. Marris for statistical advice and help, and Dr. R. A. Dale, consultant clinical biochemist, West Middlesex Hospital, for all the biochemical data.

Requests for reprints should be sent to Dr. J. S. Stewart.

\section{References}

Cossart, Y., et al. (1969). British Medical fournal, 3, 755.
Farrow, L. J., et al. (1970). British Medical fournal, 2, 693.

Fulford, K. W. M., et al. (1973). Lancet, 1, 1470.

Hersh, T., et al. (1971). New England fournal of Medicine, 285, 1363.

Krugman, P., and Giles, J. P. (1970). Fournal of the American Medical Association, 212, 1019.

Malloy, H. T., and Evelyn, K. A. (1937). Fournal of Biological Chemistry, 119,481 .

Marsh, W., Fingerhut, B., and Kirsch, E. (1959). Clinical Chemistry, 5, 119. Mirick, G. S., and Shank, R. E. (1959). Transactions of the American Clinical and Climatological Association, 71, 176.

Mohun, A. F., and Cook, I. J. Y. (1957). Fournal of Clinical Pathology, 10, 394

Reed, W. D., ot al. (1973). Lancet, 2, 690

Ross, C. A. C., and McMichael, S. (1970). Lancet, 2, 61.

World Health Organization (1973). Viral Hepatitis, Technical Report Series, No. 512. Geneva, W.H.O.

Zuckerman, A. J. (1972). Hepatitis-associated Antigen and Viruses, p. 164. Amsterdam, North Holland Publishing.

\title{
DDAVP in Treatment of Vasopressin-sensitive Diabetes Insipidus
}

\author{
M. K. WARD, T. RUSSELL FRASER
}

British Medical fournal, 1974, 3, 86-89

\section{Summary}

In 11 patients with vasopressin-sensitive diabetes insipidus the effectiveness of the vasopressin analogue 1-desamino-8D-arginine vasopressin (DDAVP) for controlling diabetes insipidus has been compared with that of lysine vasopressin. DDAVP in equivalent intravenous dosage has been found to be at least as potent and to have a more prolonged action, lasting 13-22 hours instead of 1-2 hours. Twice-daily intranasal DDAVP effected satisfactory control in all these patients, without side effects, and all the patients preferred this to their previous treatment. Single daily intramuscular injections of DDAVP were found to offer excellent control for any subject unable to manage intranasal administration.

\section{Introduction}

Improvements in the treatment of diabetes insipidus (D.I.) have obviated the need for injections in mild vasopressinsensitive cases by the use of either chlorpropamide (Wales and Fraser, 1971) and related drugs (Arduino et al., 1966) or lysine vasopressin (LVP) nasal sprays (Dashe et al., 1964). Neither of these treatments, however, can control the more serious cases satisfactorily. The recent synthesis of an analogue of arginine vasopressin (AVP), 1-desamino-8-Darginine vasopressin (DDAVP) (Vara et al., 1968), has produced an agent administrable intranasally which has the full antidiuretic effects of AVP with minimal vasopressor activity and scarcely any side effects in the dose range required (Anderson and Arner, 1972; Edwards et al., 1973). DDAVP has been found to have a greater antidiuretic effect and a longer duration of action than the natural hormone AVP (in man) or LVP (in some other species) and has been shown to be effective in controlling vasopressin-sensitive diabetes insipidus

\footnotetext{
Bndocrine Unit, Department of Medicine, Royal Postgraduate Medical School, London W12 OHS

M. K. WARD, M.B., M.R.C.P., Registrar
}

(Anderson and Arner, 1972). We report here a comparison with LVP showing the more prolonged action of DDAVP, it seffective control of a wide range of cases of vasopressin sensitive diabetes insipidus on a twice-daily intranasal regimen, and its potential usefulness in controlling such cases by single daily injections when necessary.

\section{Patients}

Altogether 11 patients were studied (table I). All had previously been investigated and diagnosed as having vasopressinsensitive diabetes insipidus either alone or with associated pituitary lesions. None of the patients were satisfied with their immediate previous therapy (table I). On their current regimens all had some persisting symptoms of mild thirst, polyuria, and nocturia. Six patients had had trials with either clofibrate or chlorothiazide or carbamazepine carried out by us or at other hospitals. Five patients previously treated with chlorpropamide $250-500 \mathrm{mg}$ daily had had to have the drug withdrawn or the dosage reduced because of hypoglycaemia, and one patient had had an "Antabuse" effect with alcohol. Five patients were maintained on vasopressin tannate in oil (5 IU intramuscularly on alternate days) with or without LVP nasal spray supplementation. One patient (case 2) who had been maintained on posterior pituitary nasal snuff for 10 years had not had an undisturbed night's sleep or been free of rhinitis throughout that time.

\section{Method}

Previous therapy was discontinued (five days for those on chlorpropamide and one to three days for those receiving vasopressin preparations). Thereafter all the patients' 24hour urine volumes were measured, initially when off all previous treatment and later while on maintenance intranasal DDAVP.

Before establishing the patients on maintenance therapy a series of acute studies were performed of their diuretic response both uncontrolled and after LVP or DDAVP, either as inpatients or as outpatients. Firstly, a full diuresis was ensured by an oral water load of $20 \mathrm{ml} / \mathrm{kg}$ given over 30 minutes, after which they were urged to drink an amount at 
TABLE I-Details of Patients Studied

\begin{tabular}{|c|c|c|c|c|c|c|c|}
\hline Case No. & $\begin{array}{c}\text { Age } \\
\text { (Years) }\end{array}$ & Sex & $\begin{array}{c}\text { Weight } \\
(\mathbf{k g})\end{array}$ & Diagnosis & $\begin{array}{l}\text { Duration } \\
\text { of D.I. }\end{array}$ & Previous Treatment for D.I. & Other Replacement Therapy \\
\hline 1 & 9 & F. & 37 & Idiopathic D.I. & 2 Years & $L V P$ nasal spray, Vasopressin & \\
\hline 2 & 34 & F. & 46 & Idiopathic D.I., seronegative & 10 Years & $\begin{array}{l}\text { tannate in onl } \\
\text { Posterior pituitary extract (as }\end{array}$ & \\
\hline 3 & 38 & F. & 58 & Idiopathic D.I. & 3 Years & $\begin{array}{l}\text { Vasopressin tannate in oil, } \\
\text { chlorothiazide, clofibrate }\end{array}$ & \\
\hline $\begin{array}{l}4 \\
5\end{array}$ & $\begin{array}{l}39 \\
31\end{array}$ & $\stackrel{\mathrm{F}}{\mathrm{M}}$. & $\begin{array}{l}54 \\
73\end{array}$ & $\begin{array}{l}\text { Idiopathic D.I. } \\
\text { Cerebral sarcoidosis }\end{array}$ & $\begin{array}{l}6 \text { Years } \\
9 \text { Years }\end{array}$ & $\begin{array}{l}\text { Vasopressin tannate in oil } \\
\text { Chlorpropamide, chlorothiazide, } \\
\text { LVP nasal spray }\end{array}$ & Prednisone $2.5 \mathrm{mg}$ twice daily \\
\hline 6 & 31 & M. & 95 & $\begin{array}{l}\text { Acromegaly. Postsurgical D.I. } \\
\text { (Operation age 28) }\end{array}$ & 3 Years & Carbamazepine & \\
\hline 7 & $5 \varepsilon$ & F. & 81 & $\begin{array}{l}\text { Acromegaly. Hypopituitary; D.I. } \\
\text { (Yttrium-90 implantation at } \\
\text { age 45) }\end{array}$ & 13 Years & $\begin{array}{l}\text { Chlorpropamide, chlorothiazide, } \\
\text { LVP nasal spray, } \\
\text { vasopressin tannate in oil }\end{array}$ & $\begin{array}{l}\mathrm{T}-40.2 \mathrm{mg} / \text { day, cortisone } \\
\text { acetate } 12.5 \mathrm{mg} \text { thrice daily }\end{array}$ \\
\hline 8 & & M. & 28 & $\begin{array}{l}\text { Craniopharyngioma. } \\
\text { Hypopituitary; D.I. } \\
\text { Suprasellar cyst. (Aspiration } \\
\text { at age 7) }\end{array}$ & 5 Years & $\begin{array}{l}\text { Vasopressin tannate in oil, } \\
\text { LVP nasal spray, } \\
\text { chlorpropamide, } \\
\text { chlorothiazide }\end{array}$ & $\begin{array}{l}\text { T-4 } 0.2 \mathrm{mg} / \text { day, cortisone } \\
\text { acetate } 12.5 \mathrm{mg} / \text { day }\end{array}$ \\
\hline 9 & 19 & M. & 39 & $\begin{array}{l}\text { Craniopharyngioma. } \\
\text { Hypopituitary; D.I. } \\
\text { (Attempted surgical removal } \\
\text { at age 5) }\end{array}$ & 14 Years & $\begin{array}{l}\text { Chlorpropamide, vasopressin } \\
\text { tannate in oil }\end{array}$ & $\begin{array}{l}\text { T-4 } 0.2 \mathrm{mg} / \text { day, cortisone } \\
\text { acetate } 5 \mathrm{mg} \text { twice daily, } \\
\text { HGH twice weekly }\end{array}$ \\
\hline 10 & 32 & M. & 68 & $\begin{array}{l}\text { Suprasellar tumour. } \\
\text { Hypopituitary; D.I. } \\
\text { (Aspiration at age 15) }\end{array}$ & 17 Years & $\begin{array}{l}\text { LVP nasal spray, vasopressin } \\
\text { tannate in oil } \\
\text { chlorpropamide }\end{array}$ & $\begin{array}{l}\mathrm{T}-40.2 \mathrm{mg} / \text { day, cortisone } \\
\text { acetate } 12.5 \mathrm{mg} \text { twice daily }\end{array}$ \\
\hline 11 & 22 & M. & 74 & $\begin{array}{l}\text { Diabetes mellitus. Pituitary } \\
\text { ablation with }{ }^{\circ} \mathrm{Y} \text {. Transient } \\
\text { postoperative D.I. }\end{array}$ & 3 Months & Chlorpropamide & Insulin \\
\hline
\end{tabular}

-Italics indicate current therapy for D.I. Roman type indicates therapy previously attempted.

least equivalent to the volume of urine voided. To ensure adequate corticosteroid cover in the acute study with patients on replacement steroid therapy these patients were changed temporarily to prednisone $5 \mathrm{mg}$ three times a day and also given 5-10 mg prednisone at $6 \mathrm{a} . \mathrm{m}$. on the day of the acute studies. During the acute studies urine was collected at 15minute intervals basally until at least three collections gave urine flows within $2 \mathrm{ml} / \mathrm{min}$ of each other. Then after the administration of LVP or DDAVP 15-minute urine collections continued where possible, or otherwise half-hourly to two-hourly collections were made, until urine flow returned to baseline values. It was not possible to collect urine until the effects of DDAVP had disappeared.

For each collection period both urine flow in $\mathrm{ml} / \mathrm{min}$ and urine osmolality were determined (by an Advanced osmometer). The duration of effect was the time taken for the urine flow or osmolality to return to basal values. In these acute studies with eight patients $1 \mu \mathrm{g}$ intravenous DDAVP was compared with $1 \mu \mathrm{g}$ intravenous LVP on different days. Also 5 and $10 \mu \mathrm{g}$ intranasal DDAVP given by a calibrated plastic tube (Rhinyl, Ferring A.B.) were compared with 20 and $40 \mu \mathrm{g} \mathrm{LPV}$ on other days. Thereafter treatment with twice-daily intranasal DDAVP was continued for three months or more and its efficacy in controlling urine flow was checked by measurements of 24-hour urine volume.

\section{Results}

ACUTE ANTIDIURETIC EFFECTS

Intravenous Administration.-The observed antidiuretic effects of intravenous DDAVP compared with those seen after LVP in the same eight patients are shown in table II. Both preparations reduced urine flow below $1 \mathrm{ml} / \mathrm{min}$ within 15-30 minutes. With DDAVP the peak effect was slightly greater than with LVP. At peak effect with DDAVP the urine flow was always under $1 \mathrm{ml} / \mathrm{min}$ and its osmolality 600 $739 \mathrm{mOsm} / \mathrm{kg} \mathrm{HO}$ as compared with $273-530 \mathrm{mOsm} / \mathrm{kg}$ $\mathrm{H}_{2} \mathrm{O}$ with LVP $(\mathrm{P}<0.001)$. The duration of action was 1322 hours for DDAVP as compared with 1-2 hours with LVP $(\mathrm{P}<0.0001)$. Neither drug caused a consistent change in blood pressure or pulse and no symptoms were reconded.

Intranasal Administration.-The observed antidiuretic effects of intranasal DDAVP (5 and $10 \mu \mathrm{g}$ ) and LPV (20 and $40 \mu \mathrm{g})$ are shown in table III and IV. Both preparations reduced urine flow within 30-60 minutes. The duration of action of DDAVP 5 and $10 \mu \mathrm{g}$ was 4-18 hours and for LVP 2-7 hours. In case 4 the patient had poor absorption of LVP and DDAVP from the nasal mucosa as poor responses were observed with intranasal dosage as compared with intravenous administration. The responses obtained in case 10 are shown in fig. 1.

Intramuscular DDAVP.- The potential usefulness of intramuscular DDAVP when for any reason intranasal administration is not possible is illustrated in fig. 2 and table $V$. In case 11 , in which diabetes insipidus had occurred after pituitary ablation by pituitary implantation with ${ }^{90} \mathrm{Y}$ (Fraser et al., 1962), the patient was given $2 \mu \mathrm{g}$ DDAVP intramuscularly. A good antidiuretic effect was obtained lasting 22 hours. Three patients were given $2 \mu \mathrm{g}$ DDAVP by intramuscular injection (table V), the effects lasting $14 \frac{1}{2}-22$ hours.

TABLE II-Peak Acute Effects of Intravenous DDAVP and LVP in D.I. Subjects

\begin{tabular}{|c|c|c|c|c|c|c|c|c|c|c|}
\hline \multirow{3}{*}{ Case No. } & \multicolumn{5}{|c|}{$1 \mu \mathrm{g} \mathrm{LVP}$} & \multicolumn{5}{|c|}{$1 \mu \mathrm{g}$ DDAVP } \\
\hline & \multicolumn{2}{|c|}{ Mean Basal Urines } & \multicolumn{2}{|c|}{ "Peak Effect" Urine } & \multirow{2}{*}{$\begin{array}{l}\text { Duration } \\
\text { of Action } \\
\text { (Hours) }\end{array}$} & \multicolumn{2}{|c|}{ Mean Basal Urines } & \multicolumn{2}{|c|}{ "Peak Effect" Urine } & \multirow{2}{*}{$\begin{array}{c}\text { Duration } \\
\text { of Action } \\
\text { (Hours) }\end{array}$} \\
\hline & $\begin{array}{c}\text { Flow } \\
(\mathrm{m} 1 / \mathrm{min})\end{array}$ & $\begin{array}{c}\text { Osmolality } \\
\text { (mOsm) }\end{array}$ & $\begin{array}{c}\text { Flow } \\
\text { (m1/min) }\end{array}$ & $\begin{array}{c}\text { Osmolality } \\
(\mathrm{mOsm})\end{array}$ & & $\begin{array}{c}\text { Flow } \\
(\mathrm{m} 1 / \mathrm{min})\end{array}$ & $\begin{array}{l}\text { Osmolality } \\
\text { (mOsm) }\end{array}$ & $\underset{(\mathrm{m} 1 / \mathrm{min})}{\text { Flow }}$ & $\begin{array}{c}\text { Osmolality } \\
\text { (mOsm) }\end{array}$ & \\
\hline $\begin{array}{r}2 \\
3 \\
4 \\
5 \\
6 \\
7 \\
9 \\
10\end{array}$ & $\begin{array}{r}8.0 \\
11.0 \\
13.0 \\
7.8 \\
8.8 \\
8.1 \\
7.4 \\
7.1 \\
\end{array}$ & $\begin{array}{r}35 \\
60 \\
45 \\
93 \\
95 \\
87 \\
105 \\
60 \\
\end{array}$ & $\begin{array}{l}1.6 \\
1.0 \\
0.7 \\
1.2 \\
0.5 \\
2.0 \\
1.6 \\
1.0 \\
\end{array}$ & $\begin{array}{l}436 \\
530 \\
273 \\
283 \\
537 \\
328 \\
451 \\
332 \\
\end{array}$ & $\begin{array}{l}1 \cdot 7 \\
2 \\
2 \\
2 \\
2 \\
2 \\
1 \cdot 7 \\
1 \\
\end{array}$ & $\begin{array}{r}9.0 \\
14.0 \\
14.2 \\
7.6 \\
10.0 \\
7.2 \\
8.2 \\
9.5 \\
\end{array}$ & $\begin{array}{r}46 \\
55 \\
51 \\
100 \\
68 \\
91 \\
66 \\
48 \\
\end{array}$ & $\begin{array}{l}0.3 \\
0.3 \\
0.6 \\
0.6 \\
0.5 \\
0.4 \\
0.6 \\
0.5 \\
\end{array}$ & $\begin{array}{l}713 \\
688 \\
600 \\
684 \\
680 \\
739 \\
664 \\
600 \\
\end{array}$ & $\begin{array}{l}13 \cdot 5 \\
16 \\
18 \\
18 \\
17 \\
22 \\
17 \\
16\end{array}$ \\
\hline $\begin{array}{c}\text { Mean } \pm \text { S.E. } \\
\text { of mean } \\
\mathbf{P}\end{array}$ & $9.15 \pm 0.85$ & $72 \cdot 5 \pm 9 \cdot 13$ & $1 \cdot 2 \pm 0 \cdot 17$ & $396 \cdot 2 \pm 37 \cdot 58$ & $1.8 \pm 0.12$ & $\begin{array}{r}9.96 \pm 0.96 \\
\text { N.S. }\end{array}$ & $\begin{array}{r}65 \cdot 6 \pm 7 \cdot 14 \\
\text { N.S. }\end{array}$ & $\begin{array}{r}0.47 \pm 0.04 \\
0.007\end{array}$ & $\begin{array}{r}671 \cdot 0 \pm 17.45 \\
0.001\end{array}$ & $\begin{array}{r}17 \cdot 19 \pm 0.85 \\
0.0001\end{array}$ \\
\hline
\end{tabular}


TABLE III-Peak Acute Effects of Intranasal DDAVP $5 \mu \mathrm{g}$ and LVP $20 \mu \mathrm{g}$ in D.I.

\begin{tabular}{|c|c|c|c|c|c|c|c|c|c|c|}
\hline \multirow{3}{*}{ Case No. } & \multicolumn{5}{|c|}{$20 \mu \mathrm{g}$ LVP } & \multicolumn{5}{|c|}{$5 \mu \mathrm{g}$ DDAVP } \\
\hline & \multicolumn{2}{|c|}{ Mean Basal Urines } & \multicolumn{2}{|c|}{ "Peak Effect" Urine } & \multirow{2}{*}{$\begin{array}{c}\text { Duration } \\
\text { of Action } \\
\text { (Hours) }\end{array}$} & \multicolumn{2}{|c|}{ Mean Basal Urines } & \multicolumn{2}{|c|}{ "Peak Effect" Urine } & \multirow{2}{*}{$\begin{array}{l}\text { Duration } \\
\text { of Action } \\
\text { (Hours). }\end{array}$} \\
\hline & $\underset{(\mathrm{ml} / \mathrm{min})}{\text { Flow }}$ & $\begin{array}{c}\text { Osmolality } \\
(\mathrm{mOsm})\end{array}$ & $\begin{array}{c}\text { Flow } \\
(\mathrm{ml} / \mathrm{min})\end{array}$ & $\begin{array}{c}\text { Osmolality } \\
(\mathrm{mOsm})\end{array}$ & & $\underset{(\mathrm{ml} / \mathrm{min})}{\text { Flow }}$ & $\begin{array}{c}\text { Osmolality } \\
\text { (mOsm) }\end{array}$ & $\begin{array}{c}\text { Flow } \\
(\mathrm{ml} / \mathrm{min})\end{array}$ & $\begin{array}{c}\text { Osmolality } \\
\text { (mOsm) }\end{array}$ & \\
\hline $\begin{array}{l}1 \\
2 \\
4 \\
5 \\
7 \\
8 \\
9\end{array}$ & $\begin{array}{r}13.0 \\
6.6 \\
13.0 \\
8.7 \\
5.0 \\
7.1\end{array}$ & $\begin{array}{r}53 \\
60 \\
40 \\
102 \\
73 \\
79\end{array}$ & $\begin{array}{l}1.6 \\
0.6 \\
5.3 \\
0.3 \\
0.3 \\
0.3\end{array}$ & $\begin{array}{l}296 \\
413 \\
115 \\
481 \\
717 \\
436\end{array}$ & $\begin{array}{l}1.5 \\
3.5 \\
- \\
3 \\
5 \\
3\end{array}$ & $\begin{array}{r}13.3 \\
10.1 \\
14.3 \\
9.6 \\
5.8 \\
5.2 \\
5.7\end{array}$ & $\begin{array}{r}46 \\
56 \\
48 \\
82 \\
126 \\
62 \\
125\end{array}$ & $\begin{array}{l}0.8 \\
0.6 \\
2.2 \\
1.1 \\
0.7 \\
0.3 \\
0.8\end{array}$ & $\begin{array}{l}666 \\
773 \\
315 \\
387 \\
577 \\
612 \\
796\end{array}$ & $\begin{array}{c}7 \\
18 \\
4 \\
17 \\
11^{*} \\
9 \cdot 5 \\
13\end{array}$ \\
\hline$\underset{\mathbf{P}}{\text { Mean } \pm \text { S.E. }}$ & $8.9 \pm 1.38$ & $67.83 \pm 8.89$ & $1.4 \pm 0.81$ & $409 \cdot 7 \pm 81 \cdot 65$ & $2.8 \pm 0.58$ & $\begin{array}{c}9 \cdot 1 \pm 1 \cdot 41 \\
\mathrm{~N} . S .\end{array}$ & $\begin{array}{c}78 \cdot 7 \pm 13 \cdot 2 \\
\text { N.S. }\end{array}$ & $\begin{array}{l}0.93 \pm 0.23 \\
\text { N.S. }\end{array}$ & $\begin{array}{c}589 \cdot 9 \pm 69 \cdot 06 \\
\text { N.S. }\end{array}$ & $\begin{array}{c}10.00 \pm 1.75 \\
0.004\end{array}$ \\
\hline
\end{tabular}

*Urine was not collected until effects of DDAVP had disappeared (see Method section).

TABLE IV-Peak Acute Effects of Intranasal DDAVP $10 \mu \mathrm{g}$ and LVP $40 \mathrm{\mu g}$ in D.I.

\begin{tabular}{|c|c|c|c|c|c|c|c|c|c|c|}
\hline \multirow{3}{*}{ Case No. } & \multicolumn{5}{|c|}{$40 \mu g$ LVP } & \multicolumn{5}{|c|}{$10 \mu \mathrm{g}$ DDAVP } \\
\hline & \multicolumn{2}{|c|}{ Mean Basal Urines } & \multicolumn{2}{|c|}{ "Peak Effect" Urine } & \multirow{2}{*}{$\begin{array}{l}\text { Duration } \\
\text { of Action } \\
\text { (Hours) }\end{array}$} & \multicolumn{2}{|c|}{ Mean Basal Urines } & \multicolumn{2}{|c|}{ "Peak Effect" Urine } & \multirow{2}{*}{$\begin{array}{c}\text { Duration } \\
\text { of Action } \\
\text { (Hours) }\end{array}$} \\
\hline & $\underset{(\mathrm{ml} / \mathrm{min})}{\text { Flow }}$ & $\begin{array}{l}\text { Osmolality } \\
\text { (mOsm) }\end{array}$ & $\underset{(\mathrm{ml} / \mathrm{min})}{\text { Flow }}$ & $\begin{array}{l}\text { Osmolality } \\
\text { (mOsm) }\end{array}$ & & $\underset{(\mathrm{ml} / \mathrm{min})}{\text { Flow }}$ & $\begin{array}{l}\text { Osmolality } \\
\text { (mOsm) }\end{array}$ & $\underset{(\mathrm{ml} / \mathrm{min})}{\text { Flow }}$ & $\begin{array}{l}\text { Osmolality } \\
\text { (mOsm) }\end{array}$ & \\
\hline $\begin{array}{r}3 \\
4 \\
5 \\
6 \\
10\end{array}$ & $\begin{array}{l}16.1 \\
10.0 \\
12.1 \\
10.1\end{array}$ & $\begin{array}{l}49 \\
66 \\
83 \\
70\end{array}$ & $\begin{array}{l}5.1 \\
2.0 \\
1.0 \\
1.0\end{array}$ & $\begin{array}{l}131 \\
315 \\
448 \\
411\end{array}$ & $\begin{array}{l}2 \\
2 \\
7 \\
2\end{array}$ & $\begin{array}{r}13.4 \\
13.0 \\
7.6 \\
11.1 \\
11.6\end{array}$ & $\begin{array}{l}62 \\
36 \\
83 \\
76 \\
77\end{array}$ & $\begin{array}{l}0.4 \\
0.7 \\
0.5 \\
0.8 \\
0.3\end{array}$ & $\begin{array}{l}657 \\
416 \\
692 \\
560 \\
577\end{array}$ & $\begin{array}{l}11 \\
9.5 \\
13 \\
11 \cdot 5 \\
10\end{array}$ \\
\hline $\begin{array}{c}\text { Mean } \pm \text { S.E. } \\
\text { of mean } \\
\mathbf{P}\end{array}$ & $12 \cdot 1 \pm 1 \cdot 42$ & $67 \pm 7 \cdot 01$ & $2.3 \pm 0.97$ & $326 \cdot 2 \pm 70 \cdot 86$ & $3 \cdot 25 \pm 1 \cdot 25$ & $\begin{array}{c}11 \cdot 3 \pm 1 \cdot 03 \\
\text { N.S. }\end{array}$ & $\begin{array}{c}66 \cdot 8 \pm 8 \cdot 43 \\
\text { N.S. }\end{array}$ & $\begin{array}{l}0.54 \pm 0.09 \\
\text { N.S. }\end{array}$ & $\begin{array}{c}580 \cdot 4 \pm 47 \cdot 84 \\
0.01\end{array}$ & $\begin{array}{c}11.0 \pm 0.61 \\
0.001\end{array}$ \\
\hline
\end{tabular}

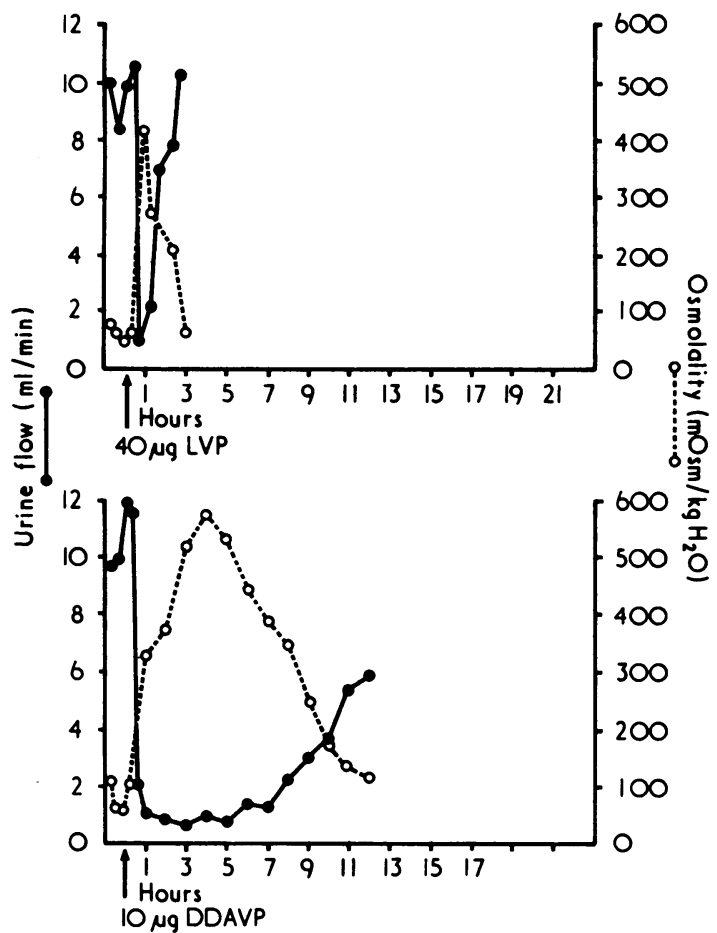

FIG. $1-$ Case 10. Effects of $40 \mu \mathrm{g}$ LVP and $10 \mu \mathrm{g}$ DDAVP given by intranasal administration.

TABLE v-Effects of Intramuscular DDAVP $(2 \mu g)$ in Vasopressin-sensitive D.I.

\begin{tabular}{|c|c|c|c|c|c|c|}
\hline $\begin{array}{l}\text { Case } \\
\text { No. }\end{array}$ & $\underset{(\mathbf{k g})}{\text { Weight }}$ & $\begin{array}{c}\text { Mean } \\
\text { Basal } \\
\text { Urine } \\
\text { (mlimin) }\end{array}$ & $\begin{array}{c}\text { Mean } \\
\text { Basal } \\
\text { Osmolality } \\
\text { (mOsm) }\end{array}$ & $\begin{array}{c}\text { Urine } \\
\text { Flow at } \\
\text { Peak } \\
\text { Effect } \\
\text { (ml/min) }\end{array}$ & $\begin{array}{c}\text { Maximum } \\
\text { Urine } \\
\text { Osmolality } \\
\text { (mOsm) }\end{array}$ & $\begin{array}{c}\text { Duration } \\
\text { of } \\
\text { Action } \\
\text { (Hours) }\end{array}$ \\
\hline $\begin{array}{r}5 \\
2 \\
11\end{array}$ & $\begin{array}{l}73 \\
46 \\
74\end{array}$ & $\begin{array}{l}6.3 \\
9.0 \\
6.4\end{array}$ & $\begin{array}{r}129 \\
87 \\
160\end{array}$ & \begin{tabular}{l|l}
0.5 \\
0.5 \\
1.0
\end{tabular} & $\begin{array}{l}851 \\
839 \\
855\end{array}$ & $\begin{array}{l}181 \\
142 \\
22\end{array}$ \\
\hline Mean & & $7 \cdot 1$ & 125 & 0.66 & 848 & 18 \\
\hline
\end{tabular}

TABLE VI-Prolonged Control of D.I. using Intranasal DDAVP

\begin{tabular}{|c|c|c|c|c|}
\hline \multirow{2}{*}{$\begin{array}{l}\text { Case } \\
\text { No. }\end{array}$} & \multirow{2}{*}{$\underset{\text { (kg) }}{\text { Weight }}$} & \multirow{2}{*}{$\begin{array}{l}\text { No Treatment } \\
\text { Urine Volume } \\
(1 . / 24 \mathrm{hr})\end{array}$} & \multicolumn{2}{|c|}{ With DDAVP Treatment } \\
\hline & & & $\begin{array}{c}\text { Dose } \\
(\mu \mathrm{g} / \text { Day })\end{array}$ & $\begin{array}{l}\text { Urine Volume } \\
(1 . / 24 \mathrm{hr})\end{array}$ \\
\hline $\begin{array}{l}8 \\
7 \\
9 \\
2\end{array}$ & $\begin{array}{l}28 \\
81 \\
39 \\
46\end{array}$ & $\begin{array}{l}3 \cdot 5-4 \cdot 5(3) \\
3 \cdot 5-4 \cdot 5(5) \\
3 \cdot 0-4 \cdot 0(4) \\
6 \cdot 9-7 \cdot 8(2)\end{array}$ & \multirow{3}{*}{$\begin{array}{c}2.5 \times 2 \\
5 \times 2 \\
5 \times 2 \\
5 \times 1 ; 2.5 \times 1 \\
5 \times 2 \\
10 \times 2 \\
10 \times 2 \\
10 \times 2 \\
10 \times 2 \\
10 \times 2 \\
10 \times 2\end{array}$} & \multirow{3}{*}{$\begin{array}{c}0 \cdot 8-1 \cdot 3(8) * \\
0 \cdot 75-1 \cdot 2(5) \\
1 \cdot 5-2 \cdot 2(5) \\
0.5-0 \cdot 8(4) \\
4 \cdot 5(1) \\
1 \cdot 5-2 \cdot 5(4) \\
2 \cdot 2-4 \cdot 2(6) \\
1 \cdot 5-2 \cdot 0(3) \\
1 \cdot 5-2 \cdot 6(2) \\
0 \cdot 7-1 \cdot 8(5) \\
0.8-1 \cdot 5(5)\end{array}$} \\
\hline 1 & 37 & 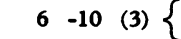 & & \\
\hline $\begin{array}{r}10 \\
6 \\
4 \\
3 \\
5\end{array}$ & $\begin{array}{l}68 \\
95 \\
54 \\
58 \\
73\end{array}$ & $\begin{array}{r}9 \cdot 5-11 \cdot 5(4) \\
3 \cdot 5-5 \cdot 0(3) \\
12 \cdot 0-15 \cdot 0(3) \\
8 \cdot 0-10 \cdot 0(2) \\
8-10(2)\end{array}$ & & \\
\hline
\end{tabular}

- Figures in parentheses are number of days of observed 24-hour urine volumes from which range is taken.

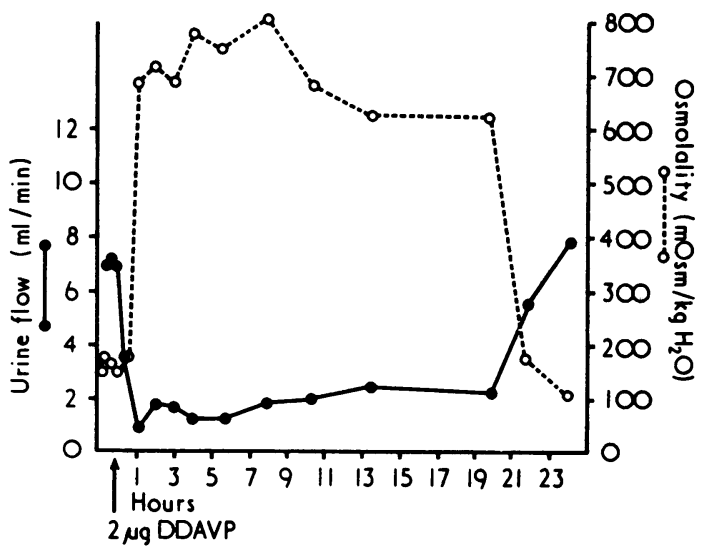

FIG. 2-Case 11. Effect of intramuscular DDAVP.

EFFECTS OF PROLONGED ADMINISTRATION OF DDAVP

As shown in table VI twice-daily intranasal administration proved an effective control of the diabetes insipidus in all our patients without any side effects being noted. During this period the rhinitis continous for 10 years after the administration of posterior pituitary snuff in case 2 completely disappeared on intranasal DDAVP. 


\section{Discussion}

Our results confirm other reports of the clinical effectiveness of this therapy (Anderson and Arner, 1972; Edwards et al., 1973) and so justify the plan to synthesize this analogue of vasopressin (Zoral and Storm, 1966). Twice-daily intranasal DDAVP completely controlled the diabetes insipidus in all our patients. Included were patients whose severe diabetes insipidus could not be controlled by chlorpropamide even when supplemented with LVP nasal spray and so had been managed with intramuscular vasopressin tannate in oil. The intranasal DDAVP method of treatment was preferred by all the patients and also achieved better control of their diabetes insipidus without any side effects.

There is no doubt that there was a need for this improvement in the replacement therapy of vasopressinsensitive diabetes insipidus. Unfortunately nephrogenic diabetes insipidus can still only be poorly controlled by the use of thiazides and restricting salt intake (Schotland et al., 1963). For mild cases of vasopressin-sensitive diabetes insipidus oral chlorpropamide (Wales and Fraser, 1971), clofibrate (De Gares et al., 1970), or carbamezipine (Braunhofer and Zicha, 1966) has been proved fully adequate. Slightly more severe cases may be fully controlled if one of these drugs is supplemented by intranasal LVP (Wales and Fraser, 1971). But hitherto for severe vasopressin-sensitive diabetes insipidus adequate control has been possible only with intramuscular vasopressin tannate in oil, whose duration of action of ten varies from inadequate shaking and warming of the phial before injection.

DDAVP is an improvement in several ways. The pure synthetic compound can be given intranasally without causing rhinitis or even the severe pulmonary reactions produced at times by vasopressin snuff (Pepys et al., 1965; Mahon et al., 1967) and years of rhinitis from this snuff may sub- side when the patient changes from the snuff to intranasal DDAVP, as our case 2 showed. Administration of accurately measured dosage can be achieved with the nasal catheter method of administration (Rhinyl, Ferring A.B.). As hoped by the synthesists (Zoral and Storm, 1966) the amino-acid substitutions involved have eliminated the pressor effects from the doses needed for replacement therapy and produced resistance to degradation so that it persists longer at the site of action (Vara et al., 1968; Edwards et al., 1973). For the occasional patients for whom intranasal administration may not be possible-for example, those with rhinorrhoea or nasal infections-DDAVP can offer the alternative control by single daily intramuscular injections.

We are grateful to Dr. Elizabeth Hughes and staff of the child health laboratory for the osmolality measurements, to Sister Kathleen Boon and staff of the metabolic unit, and to Dr. J. Mulder, research director (Ferring Pharmaceuticals), for the supply of DDAVP.

\section{References}

Anderson, K. E., and Arner, B. (1972). Acta Medica Scandinavica, 192, 21. Arduino, F., Ferroz, F. P. J., and Rodrigues, J. (1966). Fournal of Clinical Endocrinology, 26, 1325 .

Braunhofer, J., and Zicha, L. (1966). Medizinische Welt, 2, 1875.

Dashe, A. M., et al. (1964). Fournal of the American Medical Association, 190, 1069.

De Gares, J. L., et al. (1970). Annales d'Endocrinologie, 31, 300.

Edwards, C. R. W., et al. (1973). British Medical fournal, 3, 375.

Fraser, T. R., Joplin, G. F., and Steiner, R.

Mahon, W. E et al (1967). Thorax, 22, 13.

Pepys, W. E., et al. (1967). Thorax, 22, 13.

Schotland, M. G., Grunbach, M. M., and Strauss, J. (1963). Pediatrics, 31, 741 .

Vara, J., et al. (1968). Lancet, 1, 948.

Wales, J. K., and Fraser, T. R. (1971). Acta Endocrinologica, 68, 725.

Zoral, M., and Stcrm, F. (1966). Collections of Czechoslovak Chemical Communications, 31, 310 .

\title{
Thyroid Function in Patients Treated with Radioactive Iodine for Thyrotoxicosis
}

\author{
W. M. G. TUNBRIDGE， P. HARSOULIS， A. W. G. GOOLDEN
}

British Medical fournal, 1974, 3, 89-92

\section{Summary}

A series of 105 patients treated at least two years earlier with radioactive iodine for thyrotoxicosis have been surveyed. Eighty-five patients $(81 \%$ ) were euthyroid clinically and on the basis of routine thyroid function tests. Of the euthyroid patients $46(54 \%)$ had normal thyroid-stimulating hormone (TSH) levels and $39(46 \%)$ had raised TSH levels. There was no difference in serum triiodothyronine levels between these two groups but the serum protein bound iodine and serum thyroxine, though still well within the normal range, were significantly lower in the group with raised

\footnotetext{
Departments of Medicine and Radiotherapy, Hammersmith Hospital, London W12 0HS

W. M. G. TUNBRIDGE, M.B., M.R.C.P., Registrar (Presen appointment: Senior Research Assistant, Royal Victoria Infirmary, Ivewcastle upon Tyne)

TSH levels. The serum cholesterol was also significantly higher in this latter group.

Most of the euthyroid patients were seen again a year later. None had become hypothyroid and neither those with normal nor those with raised TSH levels showed any evidence of a decline in the level of serum thyroxine.

It is concluded that raised serum TSH levels in patients treated with iodine-131 are not necessarily indicative of hypothyroidism. There is no indication that patients who have this abnormality become overtly hypothyroid over a 12-month follow up.

\section{Introduction}

It is now well known that many patients treated with radioactive iodine for thyrotoxicosis eventually become hypo- 8 thyroid. This complication may occur in a relatively acute form within a few months of treatment, at which time it is not difficult to diagnose, or it may develop insidiously many $\stackrel{F}{\rightleftharpoons}$ years later, in which case it may be more difficult to recognize. ${ }^{131}$ I-treated patients evidently need prolonged and careful supervision. The recognition of hypothyroidism in such a population might seem to present no problem, but in 